\section{Structure, morphology and crystal growth of bacterial magnetite}

\author{
Stephen Mann*, Richard B. Frankel $\dagger$ \\ \& Richard P. Blakemore $\ddagger$
}

* Inorganic Chemistry Laboratory, University of Oxford, South Parks Road, Oxford OX1 3QR, UK

$\dagger$ Francis Bitter National Magnet Laboratory, Massachusetts Institute of Technology, Cambridge, Massachusetts 02139, USA

$\doteqdot$ Department of Microbiology, University of New Hampshire,

Durham, New Hampshire 03824, USA

Recent high-resolution transmission electron microscopy (HRTEM) studies of the structure and morphology of bacterial magnetite $\left(\mathrm{Fe}_{3} \mathrm{O}_{4}\right)$ crystals isolated from a magnetotactic coccus ${ }^{1}$ and from an unidentified bacterium extracted from sediment ${ }^{2}$ have shown the crystals to be well ordered single-domain particles with a morphology based on a hexagonal prism of $\{011\}$ faces truncated by specific low index planes. We report here a HRTEM study of intact magnetite crystals (magnetosomes) in the microaerophilic bacterium Aquaspirillum magnetotacticum, grown in pure culture $^{3,4}$. Our aim has been to investigate the structure, morphology and crystal growth of the magnetite particles in the light of a recent Mossbauer spectroscopy study of this organism ${ }^{\text {s }}$ which indicated, in addition to magnetite, the presence of hydrated iron(III) oxide phases together with the magnetosomes. Our results show that the mature particles are well ordered single-domain crystals of magnetite with a morphology very different from previously studied crystals and based on an octahedral prism of $\{111\}$ faces truncated by $\{100\}$ faces. We also show the first direct evidence for both crystalline and non-crystalline phases within individual magnetosomes. The results are important in aiding elucidation of the crystal growth mechanisms of biogenic magnetite.

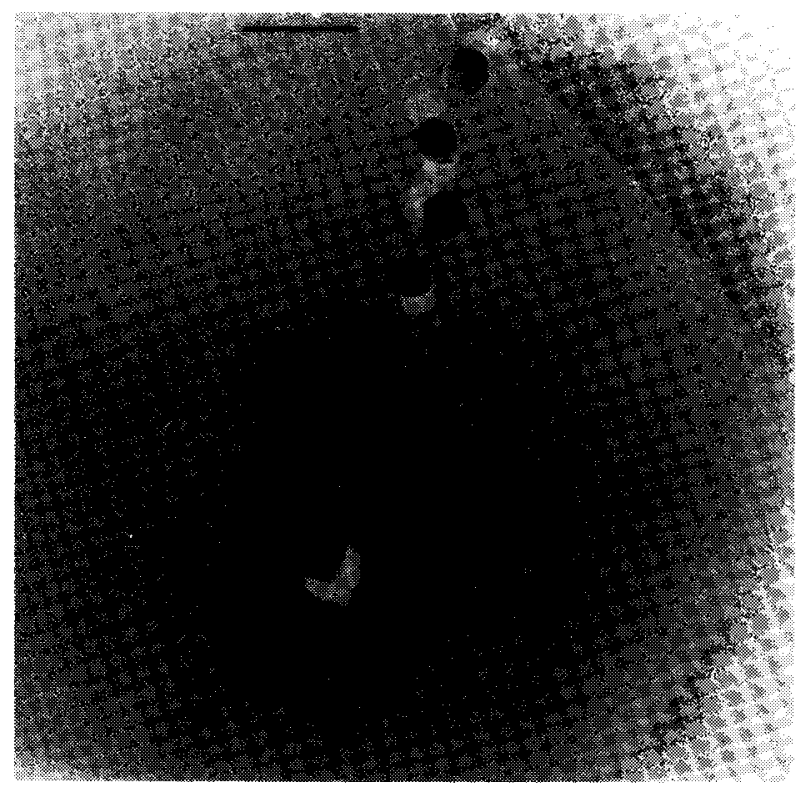

Fig. 1 Chain of magnetite particles imaged within an intact unstained $A$. magnetotacticum cell, showing a combination of large and small particles. Particle $A$ has a characteristic morphology seen in many mature crystals (see Fig. 3). Particle B is irregular in form and appears to be at a different stage in development from particle $A$. Lattice images of this particle showed crystalline and non-crystalline zones (Fig. 4). The cluster of four particles near to B was also imaged as single crystals with localized amorphous regions. They are spacially separate and therefore not a multidomain aggregate. Scale bar, $100 \mathrm{~nm}$

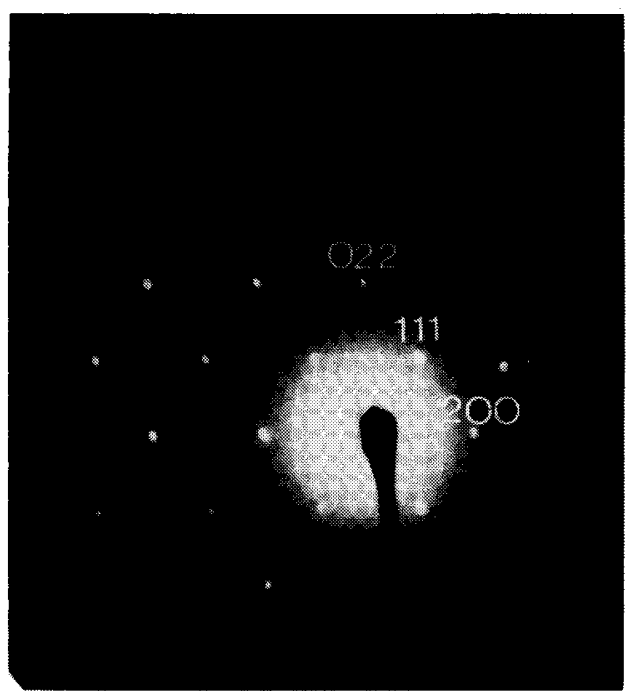

Fig. 2 Selected-area electron diffraction pattern from a mature magnetite crystal of characteristic morphology. The single crysta pattern corresponds to the $[01 \overline{1}]$ zone. The $(200)$ and $(200)$ reflec-

tions arise from double diffraction. Camera length, $115 \mathrm{~cm}$.

Cells of A. magnetotacticum are $\sim 3 \mu \mathrm{m}$ long and contain, on average, 20 intracellular enveloped magnetite particles of diameter $40-50 \mathrm{~nm}$ which are organized in a single chain that traverses the cell longitudinally ${ }^{6}$. Cells of $A$. magnetotacticum synthesize magnetite only in microaerobic conditions, accumulating $\mathrm{Fe}$ some 20,000-40,000-fold over the extracellular concentration $^{3}$. The magnetite particles are in the single magnetic domain size range and the chain of magnetosomes imparts a permanent magnetic dipole moment parallel to the cell's axis of mobility such that the bacteria orient in the geomagnetic field ${ }^{7}$.

Intact, unfixed cells were dried down onto carbon-coated electron microscope grids and investigated in a JEOL $200 \mathrm{CX}$ high-resolution transmission electron microscope operating at $200 \mathrm{keV}$ with $\mathrm{a}_{\mathrm{LaB}}$ electron source and a point-to-point resolution of $2.46 \AA$. Many cells showed chains of preferentially aligned magnetite particles of distinct morphology. Other chains were observed to contain a combination of large, well developed magnetosomes and smaller particles of irregular form, suggesting the presence within the chains of crystals at different stages of development (Fig. 1).

Crystals of distinct morphology (such as crystal A in Fig. 1) showed lattice images consistent with the cubic ( $\mathrm{Fd} 3 \mathrm{~m})$ inverse spinel structure of magnetite. The lattice images were well defined and run continuously throughout the particles, thus indicating that they are single-crystal domains. Lattice fringes corresponding to the $\{111\},\{222\},\{220\},\{200\}$ and $\{400\}$ planes were imaged and by considering the relative orientation of these fringes, the zone of projection was identified as [01i $]$. This was confirmed by selected-area electron diffraction patterns (Fig. 2). The morphology of these mature crystals was inferred from the relative directions of the fringes and crystal edges and is based on an octahedron of $\{111\}$ faces truncated by $\{100\}$ faces (Fig. $3 a, b)$. Thus, crystals imaged in the $[01 \overline{1}]$ zone often showed edges meeting at characteristic angles of $125^{\circ}$ and $110^{\circ}$, corresponding to the intersection of (100) and (111) and of (111) and (111) faces. In other mature crystals, these angles were distorted from their theoretical value; this may be a consequence of slight crystal misalignment with respect to the electron beam or it may be a real growth effect.

On the basis of the above morphology, we determined the crystal alignment in bacteria containing chains of well developed crystals. The crystals were found to be preferentially aligned, with the [111] direction parallel to the chain axis. This result is important with regard to magnetotaxis, as the easy axis of magnetization in $\mathrm{Fe}_{3} \mathrm{O}_{4}$ also lies along the [111] direction.

Our results indicate that the cellular environment in which 

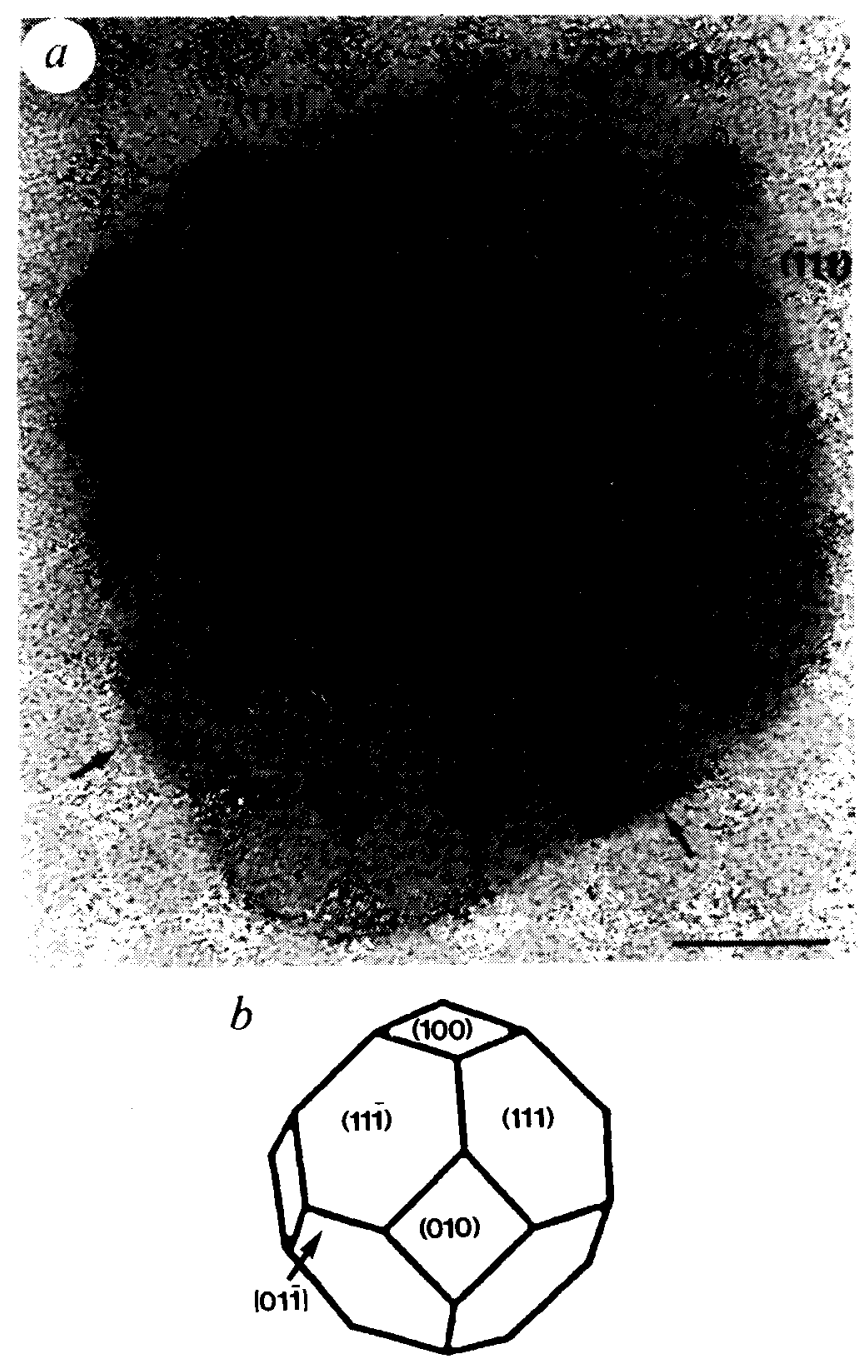

Fig. 3 a, HRETEM image of a magnetite crystal imaged in the [011] zone, showing a well ordered single-domain crystal and a characteristic morphology based on an octahedral prism of $\{111\}$ faces truncated by $\{100\}$ faces. The lattice fringes shown correspond to the $(022)$ planes and run perpendicular to the (100) face. Note that the crystal edges are not smooth and show outgrowths on the well developed $\{111\}$ faces (arrow). Scale bar, $10 \mathrm{~nm}$. $b$, Idealized morphology for biogenic magnetite crystals from A. magnetotacticum. An octahedral prism of $\{111\}$ faces is truncated by $\{100\}$ faces. Such a crystal form indicates the stabilization of the $\{111\}$ planes over other low index faces such as $\{100\}$.

the crystals grow is often well defined and influences their morphology in a way which is different from that found for other magnetotactic bacteria ${ }^{1,2}$. Many factors can influence the crystal morphology of biogenic solids ${ }^{8}$, so it may not be surprising that the morphology of the bacterial magnetites seems to be species specific. It is unclear why some mature crystals in $A$. magnetotacticum do not attain the characteristic octahedral morphology; perhaps the crystal growth processes are very susceptible to chemical fluctuations in the intra- and extracellular environments.

Lattice imaging of the irregular particles (for example, particle $B$ in Fig. 1) showed the presence of contiguous crystalline and non-crystalline regions within the magnetosome (Fig. 4). The crystalline zone was always single domain, with well ordered lattice planes of magnetite. No other crystalline phases, such as $\gamma$-FeOOH, were observed. The lattice fringes often appeared to extend into the amorphous region in a preferential direction.

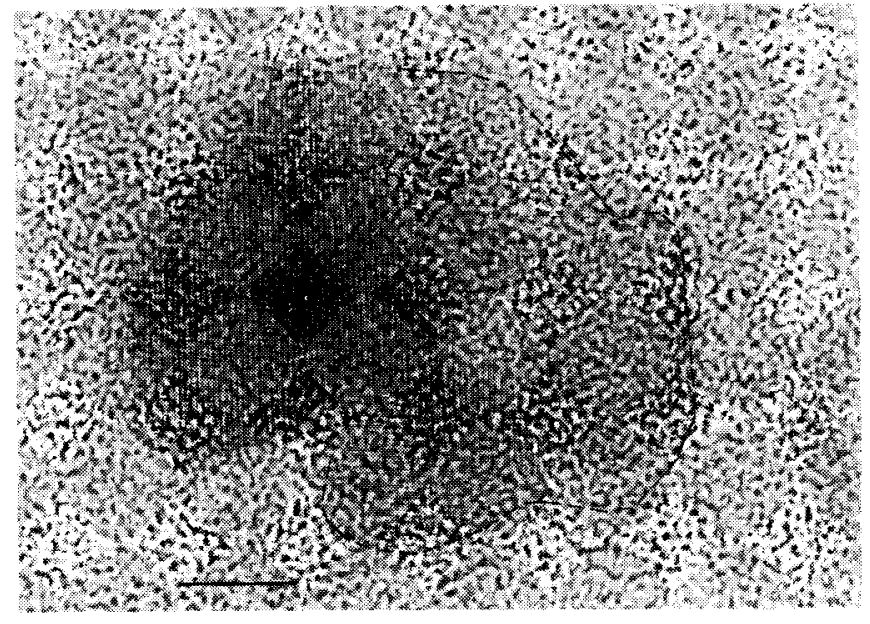

Fig. 4 HRTEM image of particle B of Fig. 1, showing the coexistence of crystalline and non-crystalline phases. The crystalline zone shows well ordered (222) lattice fringes and is a single domain. The fringes extend into the amorphous phase in a preferential direction. The superimposed black dashed line indicates the extent of the low contrast edge of the particle against the background carbon noise of the grid. Scale bar, $5 \mathrm{~nm}$.

These multi-phase particles probably represent the early stages of magnetite formation, with the non-crystalline material corresponding to the hydrated iron (III) oxide phases identified by Mossbauer spectroscopy ${ }^{3}$.

On the basis of these results, a mechanism of crystal formation can be offered. The crystallization of bacterial magnetite occurs within a localized region of the cell and involves a non-crystalline precursor of hydrated iron(III) oxide. The lattice images suggest that a growing crystal front of magnetite extends into the amorphous gel. The solid-state rearrangement could occur through a solution front at the interface of the two phases. The final crystal is single domain and often has a characteristic octahedral morphology, indicating that crystal growth is slow and that the $\{111\}$ planes are stable. Because many crystals are preferentially aligned with the [111] direction parallel to the chain axis, both nucleation and growth-processes seem to be under biological control. The origin of aqueous $\mathrm{Fe}^{2+}$ ions in the crystal growth mechanism is unclear, although $\mathrm{Fe}^{2+}$ ions have been detected in A. magnetotacticum $^{5}$. They could arise from partial reduction of the hydrated iron(II) oxide phase at the solid-state interface due to the changes in the local redox potential or they could be transported directly from the cytoplasm or periplasmic space into the crystallizing zone. The adsorption of $\mathrm{Fe}^{2+}$ ions at the hydrated iron(III) oxide surface is probably the trigger for magnetite formation ${ }^{1}$.

We thank N. Blakemore for help in preparation of the samples. R.B.F. was in part supported by the Office of Naval Research. The Francis Bitter Laboratory is supported by the NSF.

\footnotetext{
Mann S Moench, T. T \& Williams, R J P. Proc R Soc B 221, 385-393 (1984)

Matuda, Endo, J Osakabe, N. \& Tonomura, A. Nature 302, $411-412$ (1983)

3. Blakemore, R. P., Maratea, D. \& Wolfe, R. S. J. Bact. 140, 720-729 (1979).

4. Maratea, D. \& Blakemore, R. P. Int. J. Syst. Bact. 31, 452-455 (1981).

5. Frankel, R. B. Papaefthymoeu, G. C., Blakemore, R. P. \& O'Brien, W. Biochim biophys Acta 763, 147-159(1983)

6. Balkwill, D. L., Maratea, D. \& Blakemore, R. P. J. Bact. 141, 1399-1408 (1980)

7. Frankel, R. B. \& Blakemore, R. P. J. Magn. magn. Mater. 15-18, 1562-1564 (1980).

8. Mann, S. Struct. Bonding 54, 127-174 (1983).
} 